\title{
De la Reingeniería a la ReAdministración en busca de un nuevo paradigma
}

\author{
Wesley E. Bjur*
}

\section{Resumen}

El presente ensayo discute la validez de las propuestas desarrolladas por la ingenierla, concepto creado por Hammer y Champy y el de invención introducido por Osborne y Gaebler, para caracterizar las innovaciones consideradas necesarias para salir al paso a condiciones económicas que cambian constantemente; su aplicación constituye un amplio movimiento en Europa y Estados Unidos. Se parte de considerar que la reingeniería no es más que una subespecie de la idea más genérica de administración. Lamentablemente la reingenierla está seriamente limitada en su aplicación por factores del entorno, tales como tiempo (época), lugar (contexto), entorno financiero (mercado), y estructura organizacional (cultura), en consecuencia se discuten varios aspectos de la idea de un nuevo paradigma para guiar la dirección, denominado Readministración, sus elementos críticos y sus estrategias básicas.

Palabras claves:Reingenierla, ReAdministración, Paradigma Gerencial, Modelo Gerencial.

\section{From the re-engineering to the re-administration}

\section{Abstract}

This essay discusses the validity of the proposals developed by Re-Engineering, -a concept created by Hammer and Champy- and Re-Invention -introduced by Osborne and Gaebler- as characteristics necesary innovations to respond to changing economic

Fecibido: 23-11-95 . Aceptado: 30-01-96

- Master y Doctor en Ciencias Pollticas y Administración Pública. Consultor de Naciones Unidas, Banco Mundial y AID. Director del Centro de Administración Pública de la Universidad del Sur de Calfomia, E.U. Profesor invitado anualmente por la Universidad Complutense de Madrid y ol Instituto Universitario Ortega y Gasset. 
conditions, the aplication of chich represents a huge movement in both Europe and the United States. It is based on considering that Re-Engineering is a sub-species of the more generic idea of Administration. Unfortunately, Re-Engineering is seriously limited in the practice by such factors as time, place, market and structural organization (culture). In consequence it analyses several aspects of a new paradigm to guide administration, the so-called Re-Administration, its critical elements and basic strategies.

Key words: Reengineering, Readministration, Management Paradigm, Management Model.

\section{Introducción}

Este ensayo trata de la renovación de los marcos conceptuales que sostienen las teorías y prácticas de la administración/gestión contemporánea. Una renovación se ve necesaria por los cambios rápidos que se observan en elementos pertenecientes al mercado mundial, un mercado en flujo en donde las organizaciones públicas y privadas tienen que aprender a funcionar en esta última década del presente siglo.

Presiones fuertes del mercado global hacia un mejoramiento radical de la eficiencia de producción han provocado la introducción de algunas innovaciones drásticas llamadas reingeniería y/o reinvención en la estructura y gestión de los sectores públicos y privados en los Estados Unidos y especialmente en Europa, el alcance de lo cual se puede legítimamente calificar como un "movimiento".

Estos dos términos, reingeniería y reinvención han emergido como los nombres que caracterizan las innovaciones que numerosas organizaciones se han visto en la necesidad de introducir para salir al paso de las condiciones económicas cambiantes. Corporaciones grandes que producen productos y servicios para un comercio global han estado aplicando la "reingenierla", vocablo introducido por los autores Hammer y Champy (1993) en un libro enormemente popular que lleva el título Reengineering the Corporation.

Organizaciones del sector público también han sufrido el impacto de cambios políticos, sociales y económicos asociados con la reingenierla en las organizaciones comerciales. Un segundo par de autores, Osborne y Gaebler (1991). han publicado otro libro igualmente popular con el título Reinventing Government. Hay, por cierto, diferencias de énfasis entra estas dos publicaciones, a causa de diferencias en el auditorio al cual va dirigida la propuesta, sin embargo hay muchas similitudes en el enfoque $y$ en las recomendaciones que ellos hacen.

A pesar de la enorme popularidad de la fiebre por la Reingenieria/Reinvención entre consultores, ejecutivos y los que dirigen las organizaciones, algunos consultores han comenzado a cuestionar la universalidad de las formulaciones propuestas por la Reingenierla/Reinvención, especialmente la idea de aplicar un sólo conjunto de recomendaciones para remediar todo problema que se presenta en las organizaciones contemporáneas.

Después de escudriñar una literatura creciente que informa sobre éxitos y fracasos del movimiento reingenie- 
ría/reinvención, hemos llegado a creer que se ha formado su conceptualización sobre bases demasiado estrechas. Esto limita el valor de los consejos que puede proveer a consultores y gestores quienes sinceramente desean introducir mejoras modernizantes en organizaciones que no caben en las mismas categorías que los casos descritos por los autores de la Reingenierla y la Reinvención. A nuestro modo de ver, las estrategias de la reingeniería están limitadas en su aplicabilidad por factores del entorno que incluyen tiempo (época), lugar (entorno físico), mercado (entorno comercial) y cultura organizacional (entomo cultural).

\section{La Idea de Readministración}

Puesto que las presiones del mercado alectan la supervivencia de las organizaciones en todo el mundo, hay una tendencia a introducir inocentemente las mismas innovaciones que se han desarrollado en centros comerciales reconocidos como IIderes mundiales. Nuestro propósito mediante este ensayo es: a) cuestionar la aplicabilidad de algunas de las medidas defendidas por los proponentes de la reingeniería, a los contextos politico, social, económico y legal de muchos países cuyas economías también han sido alectadas por presiones similares, $y b$ ) proponer una reformulación más comprensiva y menos radical de las recomendaciones para administradores y gerentes, las cuales resultarán ser más aplicables a lo largo del tiempo, como una teoría o filosofía administrativa adecuadamente renovada.

En un intento por ensanchar la base conceptual para encarar mejor un amplio rango de problemas con los cuales luchan los gerentes contemporáneos en los sectores públicos y privados, sos. tendremos que tanto la reingeniería como la reinvención son, en realidad, subespecies de una formulación más genérica que guía la buena administración y gestión; una formulación que nombraremos, para nuestros propósitos, la Readministración, es decir, una administración renovada que incorpora principios básicos de una administración comprobadamente buena, que también incluye algunas de las estrategias novedosas que han dado buenos resultados en organizaciones que han pasado con éxito por la etapa de la reingeniería o la reinvención, principios y consideraciones administrativos "hechos nuevos" o "rehechos" para satisfacer mejor las necesidades del gerente de hoy.

Nos parece que las formulaciones de reingenieria/reinvención para la reestructuración radical de organizaciones contemporáneas, generalmente aceptadas como prácticas buenas a la luz de las presiones emanadas de condiciones reinantes en el mercado global, sưren de algunas insuficiencias potencialmente serias cuando son examinadas desde el punto de vista del "cuadro global" y de la perspectiva del largo plazo. Hemos identificado varios criterios según los cuales la estrechez de las formulaciones de la reingeniería parecen necesitar una reconsideración, éstas se resumen en la Tabla $N^{2} 1$, en la cual se comparan las formulaciones de la Reingeniería con las formulaciones ampliadas de una administración renovada, es decir, la reAdministración, que proponemos como una manera de llamar la atención acerca de las 


\section{Tabla № 1}

Contrastes entre la Reingeniería y la Readministración

\begin{tabular}{|c|c|c|}
\hline Criterio & Relngenieria & ReAdministración \\
\hline Objetivo & $\begin{array}{l}\text { Buscar ventaja competitiva, } \\
\text { satisfaciendo los clientes }\end{array}$ & $\begin{array}{l}\text { Buscar ventaja competitiva, } \\
\text { satisfaciendo tanto a los } \\
\text { clientes como a los } \\
\text { miembros de la organiza- } \\
\text { ción }\end{array}$ \\
\hline Responsabilidad Social & Ausente & $\begin{array}{l}\text { Preocupación por el } \\
\text { impacto social y ético de } \\
\text { las acciones tomadas }\end{array}$ \\
\hline Dimensión Temporal & Corto plazo & $\begin{array}{l}\text { Largo plazo, combinando } \\
\text { con acciones para corto y } \\
\text { mediano plazo }\end{array}$ \\
\hline Enfoque & Técnico & Social, técnico y ecológico \\
\hline Horizonte & Parcial Subsistémico & Sistémico, Holístico \\
\hline Filosofía de Gestión & $\begin{array}{l}\text { Revolucionaria, intenta } \\
\text { mejoras drásticas, a veces } \\
\text { aisladas del resto de la } \\
\text { organización }\end{array}$ & $\begin{array}{l}\text { Evolucionaria; admite } \\
\text { revolución dentro de un } \\
\text { enfoque sistemático y } \\
\text { programado }\end{array}$ \\
\hline Estrategia & $\begin{array}{l}\text { Un cuestionamiento radical } \\
\text { de los procesos, con } \\
\text { enfoque de "Base-Cero" }\end{array}$ & $\begin{array}{l}\text { Soluciones incrementales } \\
\text { y/o revolucionarias, } \\
\text { contextualmente definidas. }\end{array}$ \\
\hline Recursos Humanos & $\begin{array}{l}\text { Tienen que adaptarse a los } \\
\text { procesos de cambio }\end{array}$ & $\begin{array}{l}\text { Elemento esencial, } \\
\text { fundamental a los procesos } \\
\text { de cambio }\end{array}$ \\
\hline Motivación & $\begin{array}{l}\text { Tiene que ser adaptada a } \\
\text { las nuevas propuestas }\end{array}$ & $\begin{array}{l}\text { Parte integral de las } \\
\text { nuevas propuestas }\end{array}$ \\
\hline Estructura & $\begin{array}{l}\text { Nuevas estructuras con } \\
\text { menos niveles de autoridad }\end{array}$ & $\begin{array}{l}\text { Estructuras adaptativas, } \\
\text { combinadas con nuevas } \\
\text { estructuras }\end{array}$ \\
\hline $\begin{array}{l}\text { Manejo de Recursos } \\
\text { Informáticos }\end{array}$ & $\begin{array}{l}\text { Un elemento crítico, } \\
\text { esencial para mejoras } \\
\text { radicales }\end{array}$ & $\begin{array}{l}\text { Un elemento de creciente } \\
\text { relevancia, según la etapa } \\
\text { de desarrollo de la } \\
\text { organización. }\end{array}$ \\
\hline
\end{tabular}


limitaciones percibidas en la envergadura del paradigma de la Reinvención. Examinaremos con más detalle algunas de estas comparaciones en la discusión siguiente.

\section{De la \\ Relngeniería/Reinvención a la ReAdministración}

Intentaremos clarificar algunos de los puntos principales enfatizados en la reingeniería que nos parece requieren un marco conceptual más amplio, como el utilizado en la ReAdministración.

\section{- Los Objetivos de la Organiza- ción y el Horizonte Temporal}

El Programa de Reingeniería es formulado como una respuesta de supervivencia organizacional frente a presiones originadas en el mercado global, respecto al valor percibido de las mercanclas, es decir, respecto a la relación entre precio y calidad tal como son apreciados por el cliente o comprador. En el mercado global de hoy en día, los compradores en cuaiquier continente pueden tener opción a comprar mercancías producidas en varios continentes. La evaluación del comprador del precio versus calidad, determina lo que finalmente compra. Por eso, los productores de mercancías y senvicios ubicados en cualquier rincón del mundo se encuentran compitiendo con precios y calidad existentes en un mercado mundial, factores que introducen inevitables presiones del mercado tendientes a rebajar precios por calidad equivalente del producto o servicio deseado.
En este contexto global, la habilidad para satisfacer al cliente con buena calidad y bajos precios llega a ser necesaria a la supervivencia de la organización. Para poder competir con éxito en estas condiciones del mercado, la reingeniería se rinde ante las demandas imperiosas del mercado y recomienda cualquier medida que sea posible para rebajar costes y mejorar la calidad del servicio al comprador o cliente. Uno de los resultados frecuentemente observados en programas de reingeniería es el despido de empleados, puesto que los costes de mano de obra en Europa y las Américas son generalmente altos en relación a los sueldos y salarios en el Lejano Oriente, donde pueden explotar una ventaja com. petitiva en los costes de mano de obra.

Mas recientemente, algunas referencias han comenzado a aparecer en la literatura, que previenen cierta cautela con respecto a daños a largo plazo en la salud de la organización debido al despido, bajo los criterios de la reingenierla, de grandes cantidades de personal de base. Por cierto, hay ahorros inmediatos en la nómina de sueldos, pero cuando la compañla retorna nuevamente a balance positivo, se halla en la necesidad de invertir tiempo y nuevos recursos en el entrenamiento de nuevos contratados. Es mas, la experiencia y los conocimientos respecto a las operaciones de la compañía acumulados por los despedidos, han sido irreparablemente perdidos para la compañía.

La ReAdministración, prefiriendo una visión más comprensiva, tiende a enfatizar la importancia de incluir todo empleado como parte íntegra de los procesos de cambio necesarios a la supervi- 
vencia, conservando así la socialización y cultura laboral ya logradas por ellos en bien de la organización. Lo que es realmente importante para que esta tenga éxito, es un cambio de filosofía y cultura dentro de la organización hacia un aprendizaje y un mejoramiento continuo de competencias para que la compañía pueda constantemente mejorar la tasa de rendimiento en la producción de los servicios deseados por los clientes. Aportaremos más sobre este tema on una sección que trata de recursos humanos.

\section{- Asuntos de responsabilidad social}

Preocupada con la supervivencia, la reingeniería no tiene casi nada qué decir respecto al impacto ético de las acciones tomadas. Claramente, el enfoque está en lo inmediato, el corto plazo. Una vez resueltas las cuestiones de la supervivencia, tiene que encarar nuevamente asuntos de largo plazo tocantes al medio ambiente físico y social. El marco conceptual de la ReAdministración toma en cuenta las expectativas éticas de la comunidad, representadas en el medio ambiente interno y externo de la organización.

\section{- Guiones para Gerentes}

La ReAdministración, aprovechando décadas de experiencia en el manejo de una amplia variedad de organizaciones en entornos diferentes, busca incorporar estrategias más modernas en sus recomendaciones, pero esto sin negar la utilidad de experiencias y aprendizajes anteriores, es decir, sin "botar el bebé junto con el agua del baño" "Throw out the baby with the bath").

Por contraste, la reingeniería parece ser inevitablemente revolucionaria y drástica en sus recomendaciones, parece casi enorgullecerse en rechazar lo tradicional para abrazar una reestructuración radicalmente nueva del flujo de trabajo dentro de la organización. Puede haber casos en los cuales hay beneficio en la dramatización de los cambios que se tiene que hacer, (política de choque) para obligar a los empleados a que vean sus tareas en forma novedosa. Champy $(1995,53)$ cuenta una historia acerca de cómo los ejecutivos dramatizaron la im. portancia de cambios radicales introducidos en el Arizona Public Service Company (APS):

De Michele y su equipo de reingeniería se atrevieron a dramatizar el momento critico en APS con un entierro y renacimiento simbólico. En el proceso de poner todo lo pasado "en juicio", no solamente examinaron las tareas, sino también las personas empleadas para desempeñarlas. "Todos tenían que presentarse de nuevo para re-emplearse en su propio trabajo" dijo. "Tenlan que demostrar ser la persona más calificada para este empleo... Era un asunto acompañado con miedo, porque aún mi propio re-empleo estaba en cuestión.

Hay que considerar que produce trauma, frecuentemente acompañado con una pérdida en rendimiento de los empleados cuando no tienen seguridad de su posición en la organización. Se 
agudiza el problema si los ejecutivos unilateralmente introducen cambios sin una explicación plena con respecto al por qué los cambios son necesarios y qué pueden esperar de la compañía dentro del futuro inmediato y a largo plazo.

La ReAdministración prefiere evolución antes que revolución con respecto a la introducción de cambios importantes, estructurales $u$ otros. Aboga firmemente por la táctica de involucrar pronto a todos los empleados en discusiones sobre cuáles cosas requieren cambio, como también su participación en el rediseño del flujo de trabajo y asignación de tareas. Cuando los empleados están plenamente informados respecto a las razones de los cambios drásticos que tienen que hacerse, y cómo los cambios han de ser implementados, los gerentes no sólo eliminan resistencias basadas en el temor a lo desconocido asociado con los cambios, sino frecuentemente ganan un alto nivel de motivación de aquellos que sienten que han tomado parte en el diseño de la reestructuración. Estos principios, incorporados en una tecnología de desarrollo de organizaciones llamada Action Research (Investigación Activa), han sido comprobados al través del tiempo desde los años 1950 ya que dan resultados sólidos y beneficios en programas de cambio organizacional. (Para información adicional sobre Investigación Activa, véase el documento Cambiando las Organizaciones y Evaluando los Cambios Organizacionales).

\section{- Manejo de Recursos Humanos}

Es probablemente en el manejo de los recursos humanos donde uno puede ver mejor los mayores contrastes entre los marcos conceptuales de la reingeniería y la readministración. Como ya hemos indicado, la reingeniería, en la mente de muchos, es un eufemismo por la práctica de down-sizing, es decir, el despido de un número relativamente alto de empleados, con el fin de ver ahorros inmediatos en la nómina de sueldos.

A pesar del éxito aparente del movimiento de reingeniería, existen otras formulaciones utilizadas por compañías exjtosas que parecen abogar por una línea bastante diferente, una dirección casi opuesta, a seguir. En un libro reciente, los autores James Collins y Jerry Porras $(1995,3,8)$, profesores de la Escuela de Negocios de la Stanford University (California), han publicado un estudio de lo que ellos denominan "compañías visionarias" -organizaciones comerciales grandes que han aguantado las vicisitudes de la suerte de entre 50 hasta 150 años. Comenzando con una muestra de 700 compañías, estos autores redujeron su lista a 18 compañías fundadas antes de 1950, con el razonamiento de que cualquier compañia operando con éxito desde 1950, había demostrado ser más que un mero beneficiario de un gran IIder o una gran idea. La edad media de las compañias estudiadas era de 92 años de existencia. El punto clave enfatizado por los autores es que estas compañías visionarias que estudiaron muestran un notable aguante, una habilidad de rebotar adversidades que han encarado exitosamente sus operaciones durante generaciones. Su primer capítulo incluye una lista de una docena de "mitos" que son rotundamente descontados por las experiencias de estas compañías visionarias. Un mito, 
tal vez de interés de los que abogan por la reingeniería, es el siguiente:

Mito 3: Las compañías más exitosas existen primordialmente para maximizar sus ganancias financieras.

Realidad: contrario a la "doctrina" de las Escuelas de Negocios", lo de maximizar ganancias a los accionistas o la maximización de ganancias no ha sido la fuerza dominante, ni tampoco el objetivo primario al través de la historia de estas compañias visionarlas. Las compañias visionarias persiguen un conjunto de objetivos, de los cuales la ganancia financiera es sólo uno, y no necesariamente el primordial ... Ellas son igualmente guiadas por su ideología central -sus valores fundamentales, y un proposito que sobrepasa la mera ganancia, el lucro. Sin embargo, paradojicamente, las compañias visionarias han ganado más dinero que las otras, las cuales, en comparación, se declaraban dedicadas a las ganancias como principal propósito.

A lo largo de su libro, los autores reiteran constantemente un temario dual: "Preserve the core and stimulated progress" (Preservar el núcleo y estimular el progreso), frase que es identificada como la esencia de la compañía visionaria. EI éxito de estas organizaciones, afirman los autores una y otra vez, nace de procesos subyacentes y una dinámica fundamental, que están enclavados en la vida cotidiana de la organización. No depende de algún líder brillante o visionario, ni tampoco de alguna gran idea incorporada en la fundación de la firma.
Una de las compañlas considerada como ejemplar es la Jhonson \& Jhonson, fundada en 1886 para fabricar productos médicos, con énfasis especial en vendas antisépticas para cirugías y aplicaciones médicas. La filosofia de la compañla Jhonson \& Jhonson, pone como primera prioridad servicio al cliente; servicio a los em. pleados y gerentes, figuran en segundo y tercer lugares y responsabilidad con los accionistas figura como quinto y último.

Leyendo este estudio de compañías visionarias, uno nunca encuentra ejemplos de despido de empleados en grande escala. A la verdad, cuando circunstancias externas hayan obligado cualquier despido, siempre se ha hecho con expresiones de hondo sentimiento y preocupación por el bienestar de los empleados.

Estas compañías exitosas se caracterizan por fuertes inversiones en educación y entrenamiento de sus empleados. Su dedicación al progreso a largo plazo implica el desarrollo de nuevas competencias para todos sus empleados, para que puedan adaptarse al constante tren de innovaciones que caracteriza el progreso de la organización. Estas políticas de personal crean un sentir de lealtad y confianza de parte de los empleados, lo que facilita la introducción de cambios potencialmente difíciles de acomodar.

\section{La Idea de Aplicar Reingeniería en la Corporación}

Como ya hemos dicho, la idea y práctica de la reingeniería en la corporación ha llegado a ser el nombre contem- 
poráneo de una revolución verbal en la gestión que ha barrido Europa y las Américas en esta última década. Como evidencia del alcance de esta revolución, Champy $(1995,2)$ cita un estudio hecho en la primera mitad de 1994 por la SCS Index (una sección de la firma consultora que el Sr. Champy encabeza) acerca de 621 compañlas seleccionadas entre 6000 de las más grandes corporaciones en Europa y Norte América. De 497 compañlas norteamericanas, $69 \%$ reportaron que ya estaban involucradas en uno o más proyectos de reingenierla y de 124 compañías europeas, $75 \%$ reportaron datos similares. La mitad del resto de los encuestados reportaron que estaban contemplando tales proyectos. El libro de Hammer y Champy (1993), Reengineering the Corporation, ha vendido casi dos millones de ejemplares alrededor del mundo desde su publicación, llegando a constituirse on el "manual" semioficial para aquellos gerentes que desean introducir cambios dramáticos para mejorar la capacidad competitiva de sus compañías.

Sin embargo, en un segundo libro, Champy (1995) admite con cierta reluctancia, que "la reingenierfa está en dificultades". Escribe que a pesar de las historias dramáticas de éxitos, "las recompensas parecen haber resultado muy cortas respecto a su potencial" $\theta$ identifica como obstáculo la gestión. Esto lo hallamos interesante e importante, puesto que hasta hace poco el entoque mayor del movimiento de la reingeniería ha sido el mejoramiento de los procesos de productividad dentro de la compañía, no sobre los gerentes ni tampoco en el personal que ellos dirigen.
Es mas, Champy $(1995,19)$ reporta que los ejecutivos y administradores han sufrido fuertes despidos desde que los proyectos de reingeniería han comenzado a implementarse. El número de despidos de administradores durante los últimos cinco años suma la figura asombrosa de 1,4 millones de ejecutivos, gerentes y administradores profesionales, comparados con 782000 despidos de dirigentes desde 1981 a 1986. Esta figura (que no toma en cuenta un gran número de renuncias presentadas más o menos obligatoriamente, ni tampoco la pérdida de centenares de millares de empleos borrados de los organigramas de la nación), suma un término medio de 23000 empleos gerenciales por mes, o sea, 133 cada hora hábil de trabajo.

\section{La prueba Contemporánea de los Gerentes}

Champy marca cuatro olas o fases de esta reducción a gran escala de per. sonal administrativo. La primera ola nació de la necesidad de reducir costos para poder seguir operando. La segunda se presentó con el desafío de que los administradores no añaden valor comercial a los productos y/o operaciones. La tercera llegó con el reconocimiento que los gerentes en niveles medios hacen poco más que juntar y mover datos dentro de la organización. La cuarta ha venido con el mismo movimiento de la reingenieria.

Mientras que el libro de Hammer y Champy (1993) se dedicaba mayormente a la reingeniería del trabajo -de los procesos operacionales en la cadena que agrega valor al producto- Champy, en su se- 
gundo libro (1995), declara que propone dedicarse a la gestión y que está escribiendo para gerentes. Reconoce que la revolución administrativa ahora en progreso requiere una nueva definición de quiénes pueden considerarse gerentes, porque en organizaciones donde se ha aplicado plenamente la reingeniería, la responsabilidad y la autoridad son tan ampliamente dispersadas a través de la organización que casi todo empleado puede ser considerado un gerente, por lo menos de su propio trabajo. Pero aún cuando la jerarquia haya sido muy reducida, todavía quedan vestigios del sistema de autoridad jerárquica que se pueden discernir en diferentes niveles de responsabilidad administrativa:

1) Gerentes de símismos, responsables sólo por la calidad de su propio trabajo;

2) Gerentes de procesos y personal, que responden por el trabajo de otros individuos, un equipo, o un grupo de equipos que trabajan mano a mano con los clientes;

3) Gerentes expertos, cuya responsabilidad es el cuidado y desarrollo de la inteligencia de la compañía;

4) Gerentes de la firma, CEOs (Chief Executive Officers), jefes de división, todo aquel que tenga responsabilidad sobre las ganancias y pérdidas.

Estas nuevas condiciones, según Champy, dan una perspectiva completamente nueva con respecto a ideas de responsabilidad y autoridad gerencial, ideas que, en teorías anteriores, fueron asignadas casi todas a los niveles administrativos de la organización. Antiguas concepciones respecto a jerarquias de autoridad basadas en su colocación en el organigrama están desechadas. Lo que vale -por poderio, autoridad, responsabilidad-es aquello de lo cual uno es capaz, tu mismo, tu con tus propias competencias $y$ cualidades personales, escribe Champy $(1995,22)$.

\section{Rechazo del Modelo de Máquina}

Hay fuertes críticas de la metáfora de la máquina para explicar acciones y actividades humanas on estas nuevas formulaciones. Champy $(1995,20)$ escribe que la principal debilidad de la metáfora de la máquina era que promovía una cultura de burocracia, es decir, un ambiente moral que estaba enfocado sobre actividados antes que resultados. Además de la máquina, Champy menciona otras metáforas a veces utilizadas para caracterizar las organizaciones. Estas incluyen números ("the bottom linel), fuerzas (como en "fuerzas de mercado"), poder (como en "el poder ejecutivo"), cosas (como "el producto") y recursos humanos (como en "nuestros empleados"). Sin embargo, ninguna de estas metáforas ofrece gran iluminación para explicar la crisis percibida en las organizaciones y sus gerentes; tampoco proveen guías respecto a lo que se tiene que hacer para sanear los problemas que cada día son más notables.

\section{Un Entorno de Miedo}

A medida que los empleados reconocen las amenazas inherentes en proyectos de reingeniería, un miedo perverso de pérdida de empleo comienza a de- 
gradar las relaciones de trabajo dentro de la organización. Champy $(1995,49)$ lamenta que el capitalismo es un sistema que literalmente funciona a base de temor. Si es verdad, esto implica que los niveles ejecutivos encaran un desafío enorme por crear un ambiente de confianza entre los empleados frente a un contexto de temores generalizados: temores de recesión económica, temores de competidores, temor de cambios políticos, temor de despidos, temor de evaluaciones negativas del jefe, temores de docenas de factores fuera del control posible de uno mismo, que puedan resultar en pérdida de empleo y deterioro del nivel de vida.

\section{La necesidad de Fundamento Moral}

Champy $(1995,54)$ escribe que es moralmente necesario proveer a los empleados algo on qué afirmarse, algo que no va a cambiar durante el proceso de reingeniería. Propone que tal vez los únicos elementos básicos disponibles para los gerentes después de la reingeniería sean los valores comunitarios de antaño, que son la confianza, integridad, imaginación y un espiritu de cooperación, los mismos de que escribió Chester Barnard en 1938.

Respecto a éstos, las reglas son inflexibles: el ejecutivo tiene que vivirlos cada dia para poder comunicarlos a subordinados y empleados. Empleados temerosos son hipersensibles a cualquier sospecha de hipocresia y/o cinismo en las relaciones superior/subordinado, to que puede hacer casi imposible la crea- ción de confianza en la integridad del ejecutivo.

Desafortunadamente, el paradigma que subyace a las prácticas empleadas en la reingenieria no ofrece una palabra respecto a estos valores venerables. En su recomendación a los gerentes encarados con proyectos de reingeniería, Champy $(1995,79)$ escribe acerca de dos familias de valores: valores del trabajo $y$ valores sociales. Los valores sociales son idénticos con los valores humanos que son enseñados $y$ alentados por padres, profesores, amigos y conciudadanos. Incluyen aquellos de sinceridad, confianza y lealtad, respecto de sí mismo y del projimo, cooperación, responsabilidad, disponibilidad de juzgar y ser juzgado. Frente a las debilidades mencionadas en el paradigma del cual nace la reingeniería, es ampliamente reconocido que se necesita un paradigma nuevo para guiar los ejecutivos en mares que todavia carecen de carta de navegación.

\section{La Idea de un Paradigma Nuevo}

Modelo, marco conceptual, paradigma, estos términos se presentan comúnmente en la literatura contemporánea para nombrar un marco de referencia, o conjunto de conceptos que gulan percepciones mentales de lo que estamos observando; ideas acerca de cómo los elementos físicos del mundo funcionan unos en relación con otros, y cómo debemos responder a lo que observamos en derredor. Tal vez una situación nos ayudará a comprender lo que significa la idea de paradigma y del conjunto de con- 
cepciones respecto al mundo físico que son enmarcadas por un paradigma.

\section{El poder de los Conceptos Paradigmáticos}

Imaginemos a Ken, un californiano con titulo universitario en ingenierfa, a quien vemos parado con su amigo Malú, sobre un promontorio de una de las islas del grupo Melanesia en el Océano Paclfico. Ambos están observando una puesta del sol. En la distancia oímos el sonido distante del oleaje y de aves marinas.

Idénticos estímulos llegan a los ojos, oídos y mentes de ambos observadores, pero la comprensión de Ken con respecto a lo que observan difiere de aquella de Malú. Malú comenta, el sol desciende al mar, para levantarse nuevamente mañana. Ken, pensando que va a educar a su amigo con respecto a cómo son estas cosas realmente, le explica que la tierra gira hacia el este con velocidad de aproximadamente 1600 kilometros/hora, dando la apariencia de que el sol se pone al oeste. Malú, no sintiendo ninguna sensación de movimiento giratorio, sonríe, pero sin creer la explicación de su amigo acerca de la puesta del sol.

¿Cómo es posible que dos personas con percepciones normales puedan tener comprensiones tan radicalmente diferentes con respecto a Lo-que-está-ocurriendo (LOEO)? La forma contemporánea de referir maneras radicalmente diferentes de explicar o comprender LQEO es utilizar el concepto de paradigma, un término usado para nombrar concepciones subyacentes con respecto al funcio- namiento del mundo físico y acerca de cómo deberian ser interpretados y comprendidos la conducta, los gestos y el hablar de las personas.

A Ken, nacido y educado en California, le han enseñado desde su infancia la versión occidental respecto a cómo está organizado el cosmos. Las categorías lingülsticas que él utiliza cotidianamente se han derivado de esta versión occidental de la llamada ciencia positiva, y con ellas Ken interpreta el mundo flsico, los seres humanos y los conocimientos relacionados con el mundo físico. Los objetos fisicos que pueblan su mundo californiano son todos comprendidos $y$ utilizados según estas comprensiones "cientficicas" que a Ken le dictan cómo es el mundo, y por lo tanto, las reglas de uso apropiado de aquellos objetos físicos.

A Malú, criado en la cultura de su Isla, lo han enseñado en forma muy dlferente en cuanto a la organizaclón del cosmos $y$ las reglas para vivir sanamente en ol mundo.

Las costumbres con funciones rellgiosas importantes en la vida melanesiana no son claramente separadas de aquellas con injerencia económica, social, polltica, recreacional, o médica. Los centros de reuniones son tanto relliglosos como sociaies; los festivales sirven objetivos religiosos y también constituyen maneras de competir por prestigio y poder politico. Ritos para el jardín, la caza, la salud y la guerra son conducldos por aquelios que están famillarizados con las formulas y encantos por virtud de sus derechos de propletarios de ellos. No hay oflcio ni sacerdote res- 
ponsable por el liderazgo en la práctica de toda, o siqulera una mayoría de los ritos más importantes. Sin sacerdocio organizado, la cultura melanesiana tampoco tiene cosmogonias o cosmologías elaboradas.

Hay creencias sobre la existencla de un sin número de duendes vagamente antropomórilcos, qulenes se ofenden tácllmente y castigan a los transgresores con enfermedad o locura. Sujetos a control por encantos o tórmulas máglcas, algunos duendes son capaces de servir al hombre para propositos tanto buenos como malos: protegiendo jardines de ladrones, rindiendo armas más mortfferas y guerreros invencibles, produclendo o cortando lluvias, sanando entermedades, evocando el afecto del otro, como tamblén destruyendo la cosecha del enemigo, sus fortunas y vidas. Además, hay espiritus de los difuntos que creen vivir en sus proplas comunidades. Frecuentemente el clan tlene su propla aldea de espiritus a donde van los difuntos y de donde emanan las aimas de los recién nacidos. (Británica, 1969, Vol. 15, 122).

Dadas estas dramáticas diferencias en las concepciones subyacentes con respecto de las creencias en cuanto a la organizaclón del mundo en que se vive y acerca de cómo funcionan los elementos de la vida cotidiana, es fácil entender cómo estfmulos visuales y auditorios idéntlcos pueden ser interpretados en forma radlcalmente diferentes por Ken y Malú. Puesto que el mismo evento es Interpretado diferencialmente, es predecible que cada uno analizará y responde- rá viendo un repertorio diferente de opciones consideradas apropiadas al estímulo.

Un colega a quien admiro una vez me comentó: Si la gente llega a ver al mundo con ojos diferentes, su conducta cambiará como respuesta al nuevo mundo que ven. Aprender a ver el mundo con ojos diferentes se llama cambiar de paradigma, lo que significarla cambios importantes en los conceptos asumidos con respecto a cómo funciona el mundo, como también cuáles son las respuestas apropiadas a la luz de este nuevo entendimiento acerca de cómo funcionan realmente las cosas.

\section{Algunos Elementos de un Nuevo Paradlgma para Gerentes}

Los que abogan por la "reingenierla" de la corporación, y/o la "reinvención" del gobiemo, han enfatizado fuertemente la necesidad de cambios radicales en la manera en que las organizaciones comerciales y públicas son conceptualizadas, organizadas y administradas. Hammer y Champy (1993), en el libro Reeng:neering the Corporation, declaran que la fórmula propuesta por Adam Smith para la especialización y división del trabajo ya no es válida como principio que sostiene el diseño y organización de los procesos productivos. Osborne y Gaebler (1991), en Reinventing Govermment, terminan su libro con una llamada a "un cambio en el modelo básico de gobierno utilizado en América. $Y$ junto con ellos, un número importante de autores de literatura gerencial contemporánea declaran la necesidad urgente de desarrollar un conjunto 
radicalmente nuevo de concepciones subyacentes con respecto a: que hay que hacer y cómo hacerlo. (Hammer \& Champy, Collins \& Porras, Champy, Chin-Ning, Senge, Goldratt \& Cox).

\section{¿Qué diferencias hay para los Gerentes Hoy en Dia?}

Algunos de los factores que parecen sostener el argumento para desechar las maneras tradicionales de gerenciar, en favor de un marco conceptual revisado para dirigir las organizaciones y su trabajo productivo, son los siguientes:

1. La competencia mundial, entre proveedores múltiples, que reemplaza arreglos tipo cartel que proteglan territorios y/ proveedores monopolisticos de ciertos productos o servicios para ciertas regiones o poblaciones.

2. Reglas del juego empresariales que reemplazan arreglos burocráticos tradicionales en las estructuras de autoridad dentro de las organizaciones.

3. Las racionalidades tipo economia/ingenierla reemplazan las ciencias de la conducta, las llamadas ciencias blandas, con respecto a la contratación y gestión de recursos humanos.

4. En el trabajo, equipos autónomos de trabajo reemplazan sistemas jerárquicos de autoridad representados por las relaciones tradicionales entre supervisor y subordinado.

5. La responsabilidad compartida para todo aspecto operacional reemplaza la responsabilidad según la norma, en donde una descripción de tareas especificaba, en detalle, las responsabilidades del empleado.

\section{Concepciones Subyacentes del Paradigma de la Relngenlería}

Los conceptos de fondo que guían el pensar de aquellos que abogan por la reingeniería como santo remedio para los problemas organizacionales de hoy, parecen surgir de las siguientes proposiciones:

1. El mundo en que vivimos es un gran mercado, vasto y competitivo, en donde los empresarios luchan entre si para crear, producir, y vender productos $y / 0$ servicios a consumidores $y$ clientes más y más sofisticados.

2. Las materias primas disponibles hoy en el mundo, pueden ser transtormadas por la ciencia y la tecnologla aplicadas, en productos juzgados necesarios y útiles por consumidores suffcientemente sofisticados para hacer juicios comparados con respecto a calidad y precio en relación a lo que quieren y pueden desembolsar.

3. La multiplicidad de productos y la mejorada sofisticación de los compradores, significa que los factores de precio y calidad son evaluados constantemente, mientras el comprador decide comprar o no comprar. Estos factores imponen una presión constante sobre los productores para mejorar la calidad y rebajar el preclo y colocan al cliente en una posiclón de mucho más poder que en décadas anteriores. Por lo tanto, las firmas tienen una obligación de estar más orientadas hacia el cliente y sus deseos, si quieren sobrevivir.

4. La manera de la ingenieria de produ- 
cir productos de alta calidad a bajo costo es por medio de la especialización del trabajo, la tecnificación del personal y la atención constante al uso eficiente de los recursos invertidos en operaciones.

\section{Nuevos Guiones para los Procesos de Producción}

Los proponentes de la reingeniería/reinvención declaran que la manera tradicional de entender cómo están organizados los procesos de producción, basados sobre la conceptualización de Adam Smith, publicada hace más de 200 años, ya no tiene validez. A su vez, ella produjo la formalización de estructuras organizativas denominadas por Max Weber burocráticas. Los reingenieros/reinventores proponen que el paradigma antiguo de la burocracia sea reemplazado por el paradigma empresarial, lo que, a su modo de ver, provee a los gerentes con una visión superior con respecto a cuáles objetivos deberian buscar y lo que tiene que hacerse para lograr las eficiencias requeridas por el mercado global de hoy.

Es asi que el marco conceptual tradicional utilizado por los gerentes para entender a) cuál es el problema básico, y b) cuáles reglas deberían guiarla toma de decisiones, es criticado como inapropiado a la luz de lo que está ocurriendo en la gestión de las organizaciones tanto públicas como privadas. Las respuestas a nuevas condiciones del mercado y las innovaciones que están introduciendo, no corresponden a las formas tradicionales, burocráticas, para analizar y remediar problemas, si no nacen de una perspectiva empresarial. Por lo tanto, ellos alegan que un cambio de paradigma gerencial está ocurriendo en nuestro tiempo. Los ejecutivos que vislumbran el futuro piensan como empresarios, no como burócratas tradicionales.

\section{Apoderamiento versus Responsabilidad según la Norma en Sistemas Autoritarios}

Los promotores tanto de la reingeniería como de la reinvención abogan enérgicamente por el abandono de las formas burocráticas tradicionales de autoridad jerárquica, con sus niveles de supervisores y gerentes, a favor de equipos de trabajo y el apoderamiento del empleado individual para tomar decisiones al instante. Una proporción significativa de las economias reportadas en proyectos de reingeniería se debe al despido de gerentes en los niveles medios y de su cuerpo de apoyo, elementos considerados innecesarios en el apoderamiento de responsabilidades decisorias por parte de los miembros de equipos de trabajo.

\section{Problemas Encarados con Discreción Expandida}

Hemos observado que muchas de las economias logradas por la reingeniería proceden de la reducción de niveles de gerentes, basada en la noción de que la supervisión inmediata no es necesaria ni deseable cuando los mismos equipos de trabajo han sido apoderados para tomar decisiones relativas a su trabajo. Os- 
borne y Gaebler (1991) activamente abogan por la idea que los gerentes de las organizaciones que proveen servicios públicos sean autorizados a actuar como empresarios, con la libertad de forjar contratos con entes del sector privado u otros entes públicos.

Preocupaciones con respecto a este apoderamiento han provocado el cuestionamiento acerca de la reconciliación de esta discreción administrativa con las reglas de contabilidad pública, cuando apoderamiento significa libertad del administrador de hacer lo que le parezca apropiado al caso. Temen que abre la puerta a oportunidades de corrupción. Por supuesto, habrá siempre manuales y declaraciones de políticas a seguir, pero el objetivo del apoderamiento es ampliar el campo de discreción del gerente, asumiendo buena fe, integridad y responsabilidad de su parte. Tratándose del erario público, sin embargo, los contribuyentes necesitan la seguridad de que el gerente esté actuando conforme a los intereses públicos en forma más amplia y argumen$\tan$ que esto no se debiera olvidar en el apuro de relajar controles burocráticos. (Beth \& Robert, 1994,39). Claramente, algunas de las recomendaciones de esta nueva filosofía de reinvención necesitan una reconciliación con requerimientos culturales y legales que existen en diferentes paises.

\section{El Cuadro más Ampllo: Competencla versus Monopollo o Cartel}

El asunto de un productor monopolico versus competencia entre proveedo- res es común a los dos sectores, público y privado; tal vez es más verídico en Europa que en los Estados Unidos. Para las empresas de los EE.UU., la competencia del mercado es un hecho inevitable; los monopolios no son una opción. Para el gobierno como proveedor, los autores Osborne \& Gaebler (1991) abogan por el abandono de los monopolios legalizados, a favor de una búsqueda de economlas de eficiencia incentivadas por la competencia entre proveedores. Ambos sectores son motivados as a rebuscar un nuevo paradigma gerencial en donde la motivación hacia la eficiencia y calidad es optimizada vía mecanismos de licitación. Ambos presumen que hay necesidad de optimizar el uso de la tecnología disponible en este empuje sin fin para mejorar la eficiencia productiva.

\section{El Uso de Contratistas Prlvadas en el Goblerno Relnventado}

Según Osborne \& Gaebler (1991), la aplicación de la idea de usar licitación entre varios candidatos a proveedores de servicios gubernamentales procedió en forma diferente que en los programas de reingenierla en corporaciones comerciales, sin embargo, el empuje hacia eficiencias económicas para el sector público tiene mucho en común con el impacto de la competencia global para el sector comercial.

Osborne \& Gaebler declaran que ol asunto de más envergadura que encaran los administradores públicos hoy no es 10 público versus lo privado, sino monopollo versus competencia. La fuerza motor de 
esta innovación conceptual en las politicas públicas ha sido una rebeldia de parte de los contribuyentes que ha repercutido en bajas importantes en el total de contribuciones. Pese a ello, los ciudadanos todavla demandan el mismo nivel y calidad de servicios públicos que antes. Esto ha inspirado muchisimas innovaciones de parte de millares de administradores de gobiernos locales para introducir nuevas eficiencias de recursos limitados a fin de proveer un máximo de senvicios a costos rebajados. El abandono de ideas tradicionales que consideraban al gobierno como el proveedor monopólico de muchos servicios públicos y la licitación de contratos entre varios proveedores como la forma básica de rebajar costos sin rebajar calidad, llegó a ser la nueva sabidurla, la reinvención de la acción gubernamental, si se quiere.

La lógica tras la idea de reinvención aplicada a agencias de servicio público, sustituye al monopolio legalizado en el cual el gobiemo es el único proveedor autorizado, por la licitación y contratos por servicios con entes particulares. El camblo radical introducido asi en políticas de administración para gobiemos locales, indica la aceptación de la lógica que cuando escasean los recursos, la competencia entre proveedores rebaja los costes e introduce eficiencias e innovaciones en operaciones.

\section{Una reformulación de la Adminletraclón: la ReAdminletraclón}

Dadas las circunstancias expuestas en párrafos anteriores, nuestro objeti- vo ahora es explicar por qué el movimiento de la reingenieria, corrientemente la receta más popular para el mejoramiento radical de las organizaciones comerciales, parece sufrir deficiencias cuando es aplicada como marco genérico para guiar la planificación y acción administrativa en relación a las necesidades de muchas organizaciones públicas y privadas, especialmente aquellas cuyos propósitos principales son gubernamentales y/o sin fines de lucro.

Proponemos unas formulaciones consideradas necesarias para manejar organizaciones en los últimos años del presente milenio, un esquema que hemos denominadola ReAdministración. La idea de ReAdministración (Administración Renovada) incluye unas caracteristicas ya identificadas y discutidas como elementos crftlcos de una administración buena por varias décadas. Estos son:

1. La necesidad de comprender el contexto, es decir, el entomo cultural, poIitico y legal.

2. La importancia de una misión u objetivo claramente definida.

3. Consideraciones sobre cómo son afectados los recursos humanos y su desarrollo.

4. Manejo de recursos fisicos y financieros

5. El logro de resultados sociaimente relevantes

Sostenemos que estos elementos forman la espina dorsal de todo sistema administrativo que busca éxito, y por lo tanto, serán puntos de constante preocupación para los administradores. Y como la columna vertebral es sólo una parte de la 
construcción y sostenimiento del cuerpo humano, asi también hay otros elementos, los que llamaremos estrateglas, que son igualmente necesarios a las prácticas de la buena administración, cosas que deberiamos perseguir en los esfuerzos de implementación. Algunas de las estrategias importantes son las siguientes:

\section{Estrategla 1: Perfecclonar la Capacl-} dad Diagnóstica

Es importante entrenar a empleados y administradores, a todo nivel, para que sean capaces en cuaiquier momento, de identificar y evaluar dónde está la organización en relación a dónde quiere estar. Todo esfuerzo gerencial está enfocado a eliminar la brecha percibida entre la situación actual y la deseada, como mejor juicio. Cuando esto no ocurre, los empleados hacen lo único posible: se concentran en sus tareas inmediatas, a veces hasta desconectadas del resultado productivo deseado.

Estrategla 2: Analizar el Flujo de Trabajo

Casi toda la literatura contemporánea enfatiza la importancia de una claridad amplia con respecto a la meta principal de la organización, es decir, la razón y propósito de su existencia. Cuando esta meta queda bien clara, es entonces posible analizar si acaso cada tarea observada contribuye al proposito asumido por la organización.

La ambigüedad con respecto a la verdadera meta o metas de una organización es demasiado común en la vida organizacional. Frecuentemente los planes y pronunciamientos declaran clerta cosa, mientras un análisis de las tareas en ope- ración revela poca conexión con los objetivos propuestos. otras veces, las tareas estarán bien alineadas con los objetivos, pero el flujo de trabajo es organizado en secuencia ilógica o redundante, con resultados igualmente desastrosos. Estos asuntos no son tan complejos, de modo que un administrador entrenado en organización y mé todos tenga dificultad en resolverlos.

\section{Estrategla 3: Formación de Equipos de Impacto}

La formación de equipos interdisciplinarios constituidos por personas traldas de varias unidades organizacionales puede ser la forma más sencilla y menos costosa de analizar y resolver "cuellos de botella" en procesos productivos. Rápido, porque habrá una concentración especifica sobre el problema u objetivo a resolver. Eficaz, por razón de una visión slstemática y compartida por causa de su constitución interdisciplinaria $\theta$ interfuncional.

\section{Estrategla 4: Reducción de Niveles Or- ganizaclonales}

El mundo organizacional de hoy uttliza estructuras en donde jerarqula y niveles de comando gradualmente pierden su prestigio y eficacia, porque ya no responden a la necesidad de flexibilidad y rapidez requerides por mercados constantemente en flujo y clientela más y más extgente. Estructuras "planas" -no más de tres niveles- tienen más sentido y permlten un flujo de comunicación más fácll y más directo.

\section{Estrategla 5: Rebuscar Formas Partlcl- patlvas de Direcclón}

Empleados competentes, cada 
quien a su propia manera, desean ser señores de sus propios destinos. Las organizaciones pueden contribuir a esta necesidad según el grado en que existe un sistema participativo para la formulación de metas y objetivos. Cuando los empleados sienten que están participando eficazmente en el análisis de los problemas y el diseño de las soluciones, tienden a estar más motivados para hacer mejor su trabajo a fin de garantizar el exito del programa de cambio. Esto resulta en beneficios importantes a la organización.

\section{Estrategla 6: Entrenamiento y Desarro- llo de recursos Humanos}

Es de fundamental importancia que las organizaciones ínviertan en el desa: rrollo de sus empleados. En una mayoría de casos, el empleado recientemente coniratado puede carecer de competencias relacionadas al trabajo esperado por la organización. En tales situaciones, el entrenamiento se ve como necesidad. Programas de entrenamiento también facilitan la incorporación de nuevas tecnologías sostenidas por habilidades recién adquiridas por medio de entrenamiento o educación.

Sin embargo, reconocemos que el entrenamiento a nivel de entrada no es suficiente. Es importante manifestar un interés concreto en la educación y desarrollo de empleados. Una persona en de. sarrollo trasciende conocimientos enteramente funcionales en relación a su trabajo. Adquiere además una calidad esencial: la habilidad de reflexionar, de aprender cómo aprender. Feliz la organización que cuenta con empleados poseedores de tal perfil.
Nuestra experiencia personal nos dice que mientras las personas son capacitadas, desarrolladas, son de verdad, más exigentes respecto a calidad; al mismo tiempo son más tolerantes y adaptativos frente al inicio de cambios que los afecten. ¿Acaso esto no es exactamente lo que deseamos en las organizaciones? ¿Será posible lograr organizaciones eficientes, eficaces $y$ efectivas sin empleados capacitados? Difícilmente; tal vez imposible.

\section{Estrategla 7: Centrar la Atención en el cllente}

No se puede entatizar demasiado la importancia del cliente. Sin clientes no habría necesidad de organizaciones; son la única razón tras su formación. Pero es demasiado fácil olvidar al cliente, tornando la vista hacia aspectos intemos, rindiendo una visión muy próxima a la organización, pero dejando al cliente sin lugar.

Pues, el centrar la atención sobre el cliente implica no solamente una mención verbal, sino el delineamiento de acciones especificamente entocadas a la clientela: visitarlos, investigar, escudriñar sus percepciones y recibir sugerencias acerca de cómo mejorar la calidad de los servicios que se les prestan. Los comerciantes y ejecutivos generalmente reconocen la importancia de esto, pero lo hallan difícil, puesto que la investigación de mercado es difícil y cara. Una de las me* jores respuestas con respecto a la investigación del mercado fue dada por Akio Morita, el presidente de la Sony, quien respondib: Nosotros no hacemos investigación del mercado como lo hacen en Estados Unidos. Sencillamente escucha- 
mos a nuestros clientes. No hay duda de que una administración centrada en el cliente es la "regla de oro" en la construcción de una dirección que aspira al éxito en el mundo de hoy.

\section{Conclusiones}

\section{El Paradlgma de la ReAdministración}

A través de este ensayo hemos discutido varios aspectos de la idea de un paradigma para guiar la dirección. EI tema impregna mucha literatura hoy en dla. Virtualmente todo libro que toca tomas de nuevos acercamientos a "management" enfatiza la necesidad de que gerentes culturizados en el occidente aprendan a pensar de nuevas maneras, a reconocer cambios importantes en la conceptualización de su trabajo para poder "ver" maneras creativas de reestructurar la asignación de personas, recursos y procesos de trabajo como respuesta innovadora a condiciones que exhiben cambios dramáticos.

El lenguaje empleado para hablar de esto es la idea de un cambio de paradigma, cosa frecuentemente caracterizada por un "|ah-jahl" - es el momento cuando uno de repente "ve" lo que está ocurriendo en el entorno con nuevos ojos y por consiguiente, entiende en forma diferente su realidad. Hasta hace poco, se ha creido que generalmente el "paradigma nuevo" reemplaza al antiguo, tal como la revelación Copernicana acerca de que nuestro sistema planetario gira alrededor del sol, suplantó la idea de un cosmos terracéntrico. Gerentes orientados al futuro bien pueden ser convencidos que les guslarla contemplar su mundo organiza- cional con nuevos ojos, pero ¿cómo lograr tal objetivo?

\section{Un Camblo "Fuzzy" (Dlfuso) del Para- digma}

En realidad, muy pocos de los llamados "cambios de paradigma" resultan ser tan radicales como los ejemplos comúnmente ofrecidos. Aunque necesariamente involucran cambios palpables en la conceptualización de las ideas en juego, sin embargo, sería más usual y tal vez mas correcto considerarlos como "ajustes" en nuestras concepciones con respecto al funcionamiento del mundo, frecuentemente por la incorporación de nuevos conocimientos o nueva comprensión de propiedades y mecanismos que operan en el mundo.

En literatura muy reciente, se comienza a hacer notoria la necesidad de un cambio indispensable en el modo de pensar de todo gerente culturizado en la ciencia y lógica del occidente. Se trata de llegar a trascender las limitaciones de la Logica Aristoteliana, aquella forma de pensar que busca la "única y mejor forma" de analizar y resolver problemas, que es tan característica de la forma de pensar de un ingeniero.

Durante los dos últimos siglos, la cultura occidental nos ha educado con la noción de que esta "única y mejor forma" es la manera natura/de pensar acerca del universo, que existen leyes naturales estructuradas en el mismo cosmos y por lo tanto, existe sólo "una manera correcta" para obrar conforme a estas leyes naturales. Pero esta forma Aristoteliana de lógica exclusiva, que opera excluyendo una alternativa a favor de la otra, no per- 
mitiendo ambigüedades, nos deja sin explicaciones de cómo deberíamos resolver casos auto-contradictorios, en donde claramente se observa una tensión. Por ejemplo, ¿cómo es posible reconciliar simultáneamente los elementos contradictorios incorporados en la misión de la firma visionaria que afirma: preserve the core and stimulated progress (asegurar el núcleo; estimular el progreso) sin perderse en el dilema acerca de cuándo deberían asegurar lo fundamental y cuándo estimular el progreso, acto que implica desafiar ideas tradicionales, ideas del núcleo de la ideología?

Una manera de reconciliarlos ha comenzado a aparecer en la literatura cientifica durante la última década. Es un desaflo a la lógica Aristoteliana, y es denominada fuzzy logic -lógica difusa(Kosko, 1993) en contraste a la "lógica formal" de Aristóteles. Está edificada sobre el concepto de conjuntos (sets) matemáticos. Un "set" o conjunto es cualesquiera colección de objetos clasificados como similares en por lo menos un aspecto. Los teóricos de conjuntos difusos manifiestan:

Los únicos subconjuntos del universo que no son difusos (fuzzy) son los conJuntos de la matemática clásica. Todo otro conjunto -de particulas, células, tojidos, personas, ideas, galaxias- en principio contiene elementos en grado varlado. Su pertenencia es parcial, graduada, inexacta, ambigua o incierta ... El grado de pertenencia a cualquier conjunto es un asunto de similaridades y contrastes percibidos. (Treadwoll, 1995).

Para los proponentes de la fuzzy logic, "es importante que comprendamos que lo que nos han enseñado a llamar pensamiento racional no es natural. Al contrario, 10 que llamamos pensamiento racional es una conducta aprendida, para luego ser aplicada al análisis de las experiencias vividas. Parsaye y Chignel (1988) escriben:

La lógica formal se ha desarrollado a pesar de tendencias inherentes de los seres humanos hacia la conducta emocional $\theta$ irracional fundada en cualquier cosa, menos en la lógica. Parte de la atracción a la lógica formal es que ella puede contrapesar la irracionalidad humana.

Las reglas de uso de la lógica Aristoteliana presuponen que todo puede ser definido como perteneciente a una categoría $u$ otra. No se permiten tonos de gris; ninguna admisión de "parcialmenten ni "mayormente", todo es percibido como que existe en categorías mutuamente exclusivas si es tratable por la lógica formal. Sin embargo, la mente humana puede operar en forma muy natural en esta logica difusa. por ejemplo, una manera muy común de reconciliar elementos contradictorios que confunden una explicación "lógica" es tratarlos con un sentido de humor. Regularmente reconciliamos ambigũedades y averías de logica en torno a la incongruencia, en un chiste. Yarwood (1995) escribe: 
Una actividad que ocurre a nivel informal de casi toda organización, no obstante, es capaz de esquivar al gerente típico, pero es con todo, importante al funcionamiento de la organización. Me refiero al humor de los empleados y dirigentes de la organización. Un tipo prominente de humor administrativo cae en la categoría de humor incongruo, que se caracteriza por ironía y contradicción. Asuntos comunes, vistos desde perspectivas nuevas, pueden producir resultados humorísticos; ideas y palabras yuxtapuestas pueden aparecer con significaciones absurdas y provocativas.

Algunos ejemplos del trato humorístico de incongruencias lógicas que aparecen en la literatura administrativa incluyen la Ley de Parkinson (1957) que afirma que "el trabajo se expande de manera que llena el tiempo disponible para su terminación". El Prlncipio de Peter (1969) afirma que "en una jerarquía, cada empleado tiende a ser promovido a su nivel de incompetencia", cuya lógica lleva a la conclusión que los niveles altos de burocracias antiguas son poblados por incompetentes. Parcialmente verdaderos; parcialmente absurdos; son ejemplos acerca de cómo podemos apreciar la lógica difusa en la vida cotidiana.

Cuando refiexionamos sobre las maneras "naturales" en las cuales efec. tuamos diariamente centenares de elecciones y decisiones utilizando la "lógica difusa", no debe parecer fuera de 10 común que generalmente utilizamos partes y pedazos de varios paradigmas explicativos para comprender o explicar una ex- periencia dada. Un cambio completo de paradigma puede ser difícil o imposible, pero variaciones importantes de percepción pueden resultar de cambios en algunas partes el paradigma, especialmente aquellas partes que forman las conclusiones preliminares en respuesta a las cuestiones de percepción: ¿Qué es esto? ¿Cómo funciona?

La noción de "lógica diłusa" sirve bien a lo que llamarlamos El Paradigma de la ReAdministración. Eligiendo algunos de los elementos que pertenecen al conjunto de la reingenieria, algunos otros del conjunto llamado reinvención, y algunos más de entre los conceptos y prácticas comprobadas y verificadas de la administración venerable, se crea un conjunto paradigmático de conceptos útiles, de acuerdo a factores del entorno: tiempo (época), lugar (contexto), condiciones financieras (mercado) y clima organizacional (cultura). Necesita ser ecléctico y tiene que ser flexible para ser útil en diferentes contextos organizacionales.

Collins y Porras (1994) caracterizan esto el "Genio del $Y$ " (Genius of the And) en vez de la lógica bipolar del "O" (O éste o el otro) en la cual nos hemos educado. En su estudio, generaciones de líderes de compañías visionarias, de alguna manera lograron mantenerse fieles a su comprensión de los valores fundamentales que dan identidad y carácter a sus organizaciones, y al mismo tiempo abrazaban innovaciones que, en sus principios, pueden haberse visto como alejamientos de las formas históricas de operar.

Para resumir, los tiempos on los cuales vivimos, y los retos que encara- 
mos, no nos permiten la complacencia como líderes/ejecutivos si vamos a sobrevivir. Ideas nuevas, maneras nuevas de organizar y operar y tal vez lo más importante de todo, nuevas maneras de conceptualizar los elementos que tienen que ser abrazados, valorizados, juzgados e incorporados o rechazados para que la vida organizacional pueda progresar.

Desafortunadamente, no podemos concluir este ensayo con una lista de reglas garantizadas para prometer éxito a un cierto estilo de dirección o empresa. La colección de recomendaciones que constituyen el marco conceptual denominado ReAdministración, es necesariamente "fuzzy" -difusa- No obstante, creemos que la selección de ideas y ejemplos reportados en estas líneas ayudarán al lector a descubrir aquel conjunto difuso de ideas y directrices que le funcionen para el tiempo y contexto de su organización.

\section{Referencias Bibliográficas}

Barnard, Chester (1968). The functions of the Executive, Harvard University Press, Cambridge. See especially chapter 17 on Executive Responsability.

Champy, James (1995). Reengineering Management: The Mandate for New Leadership. Harper Collins Book, N.Y.

Chin-Ning. Chu (1992). Thick Face, Black Heart: The Path to Thriving, Winning and Suceeding. Warner Books, N.Y.
Collins, James C. \& Porras, Jerry Y. (1994). Built to Last: Succesful Habits of Visionary Companies, Harper Collins Book, N.Y.

Hammer, Michael \& Champy, James (1993). Reengineering the Corporation: A Manifesto for Bussiness Revolution. Harper Collins Books, N.Y. (Este libro ha vendido cerca de dos millones de copias desde su publicación en 1993).

Kosko, Bart (1993). Neural Networks and Fuzzy Systems. Prentice Hall, 1991, Bart Kosko and Satoru Isaka, Fuzzy Logic in Scientific American.

Osborne, David \& Gaebler, Ted (1991). Reinventing Government: How the Entrepreneurial Spirit is Transforming the Public Sector. Penguin Books, N.Y.

Parkinson, Northcote (1957), Parkinsons Law and Other Administrative Studies, Houghton Mifflin, Boston.

Peter, Lawrence \& Hull, Raymond (1969). The Peter Principle, Bantam Books, N.Y.

Senge, Peter (1990). The Fith Discipline: The Art \& Practice of the Learning Organization, Doubkeday, N.Y.

Shearer, Beth \& Fagin, Robert (1994). Empowerment and Accountability, The Public Manager, Vol. $23 \mathrm{~N}^{2} 1$.

Treadwell, William (1995). Fuzzy Set Theory Movement in the Social Sciences, in Public Administration Review, Vol. 55 , Ne1

Yarwood, Dean L. (1995). Humor and Administration. A Serious Inquiry into Unofficial Organizational Comunication. Public Administration Review, Vol. 55, $\mathrm{N}^{2} 1$.

- E Bjur Ph.D. Sacramento, California, E.U. Todos los derechos Reservados. El texto original en inglés fue traducido y publicado en portugués como capítulo II, titulado Da Reingeniería $E \mathrm{Da}$ Reinvencao A ReAdministracao: A Busca de Um Novo Paradigma en ReAdminlstracao Em Acao: A Prátlca Da Mudanca Rumo Ao Suceseo, Porto Alegre, RS, Brazil, Editora AGE, 1995. Traducción al español por el autor. 